\title{
Efecto de tres niveles de la inclusión de grasa de sobrepaso sobre la ciclicidad ovárica de hatos lecheros en los Andes Ecuatorianos
}

\author{
Effect of three levels of the inclusion of excess fat on the ovarian cyclicity of dairy \\ herds in the Ecuadorian Andes
}

\section{Andrés Moscoso-Piedra amoscosop@ucacue.edu.ec 1 *, (D) Bolívar Cabrera- Córdova bcabrerac@ucacue.edu.ec ${ }^{1}$, (DD) Manuel Maldonado-Cornejo mmaldonadoc@ucacue.edu.ec ${ }^{2}$, (D) Rocío Herrera-Herrera rherrerah@ucacue.edu.ec ${ }_{-}^{3}$}

${ }^{1}$ Departamento de la Unidad de Practicas Pre Profesionales Facultad de Medicina Veterinaria Universidad Católica de Cuenca, Ecuador ${ }^{2}$ Departamento de la Unidad de Titulación Facultad de Medicina Veterinaria Universidad Católica Cuenca, Ecuador ${ }^{3}$ Departamento de Vinculación con la Sociedad Facultad de Medicina Veterinaria Universidad Católica de Cuenca, Ecuador *Autor responsable y de correspondencia: Msc Moscoso-Piedra, Andrés Leonardo. Departamento de Vinculación con la Sociedad Facultad de Medicina Veterinaria Universidad Católica de Cuenca. Av. de las Américas y Humboldt. C.P. 010101 Cuenca, Azuay, Ecuador.

\section{RESUMEN}

Este estudio se enfoca de forma diferente sobre la ciclicidad ya que evalúa el efecto de la grasa en los tiempos y estructuras de la actividad ovárica, que resultan variables estrechamente relacionadas a la eficiencia reproductiva de los hatos típicos de la región austral de región andina ecuatoriana. El uso de grasa "by pass" tiene efectos positivos en la actividad ovárica y presencia de estructuras ováricas de los animales, a pesar de que los animales no sean altamente productores. Este estudio se enfocó en evaluar el efecto de tres niveles de dietas de Grasa "by pass" (GB): GB1\% (154-175 gr), GB2\% (296-361gr) GB3 \% (475-538 gr) y un control (GB0\%); en un grupo de 60 vacas lecheras Holstein, clínicamente sanas, de 2 a 5 partos y con condición corporal de 3 a 3.5. Se analizó el tamaño y el número de ovarios como referencia de la actividad ovárica; y la presencia de folículos y cuerpos lúteos como estructuras ováricas. Se realizaron pruebas de significancia para determinar las diferencias de las variables concluyendo que la adición de grasa "by pass" adelanta la presencia de actividad ovárica, e influye en los ciclos estrales. Se confirmó también el efecto positivo de la misma sobre la condición corporal y producción.

Palabras claves: estructuras ováricas, actividad ovárica, grasa "by pass", hatos típicos.

\section{ABSTRACT}

This study focuses on cyclicity differently since it assesses the effect of fat on the times and structures of ovarian activity, which are variables closely related to the reproductive efficiency of herds typical of the southern region of the Ecuadorian Andean region. The use of "by pass" fat has positive effects on the ovarian activity and presence of ovarian structures of the animals, even though the animals are not highly productive. This study focused on evaluating the effect of three levels of "by pass" (GB) fat diets: GB1\% (154-175 gr), GB2\% (296-361gr) GB3\% (475-538 gr) and a control (GB0\%); in a group of 60 Holstein dairy cows, clinically healthy, from 2 to 5 births and with body condition from 3 to 3.5. The size and number of ovaries were analyzed as a reference for ovarian activity; and the presence of follicles and corpora lutea as ovarian structures. Significance tests were performed to determine the differences of the variables, concluding that the addition of "by pass" fat advances the presence of ovarian activity, and influences the estrous cycles. The positive effect of the same on body condition and production was also confirmed.

Keywords: by pass fat, ovarian structures, ovarian activity, typical herds. 


\section{INTRODUCCIÓN}

Los largos periodos entre partos afectan la rentabilidad de las ganaderías, provocados por estados de anestros anovulatorios prolongados (Rhodes et al., 2003), existiendo una alta dificultad para alcanzar un reinicio de la actividad ovárica (Báez et al., 2009). Para que esto ocurra, básicamente deben ocurrir dos eventos: la involución del útero y la reactivación del eje hipotálamo-hipófisis-ovario (Morales et al., 2012), asociada a la fase de culminación de la involución uterina (Palomares, 2008). El intervalo parto-primera ovulación en el ganado lechero es de los 43-45 días (Becerra et al., 2008), pudiendo llegar incluso alrededor de los 85 días debido a una involucion uterina tardía.

Las concentraciones de gonadotropinas son muy bajas al final de la gestación (Robson, et al., 2008). El desarrollo de ondas foliculares ocurre tempranamente (Kawashima et al., 2011) posterior al parto, existiendo un incremento de la FSH (De Nava et al., 2012; Báez et al., 2009; Rivas et al., 2011) a partir de los 5 a 10 días; mientras la pulsatilidad de la LH se detecta alrededor de los 10 días (Rhodes et al., 2003). Un folículo dominante comienza a crecer de 10 a 14 días posparto (Wiltbank et al. 2002) (Silva et al., 2011), provocando la primera ovulación posparto, resultado de la la liberación prematura de prostaglandina F2a por el útero y la interacción del cuerpo lúteo (Becerra et al. 2008) (Motta et al., 2011).

Los principales factores influyentes en el anestro posparto, son: la nutrición (balance energético y condición corporal), edad al parto, estacionalidad y enfermedades del periparto (Rhodes et al., 2003; Morales et al., 2012; Becerra et al., 2008; Silva et al., 2011); provocando los distintos tipos de anestro (Rhodes et al., 2003; Morales et al., 2012; Wiltbank et al., 2002).

El consumo restringido de energía afecta la actividad ovárica posparto (Espinoza et al., 2010), provocando una pérdida progresiva de condición corporal (C.C.) (Giraldo et al., 2012); aunque no es posible medir acertadamente el balance energético (Rhodes et al., 2003; Montaño et al., 2005; Hernández y Díaz, 2011). Los indicadores cíclicos reproductivos pueden ser una herramienta para cuantificar este fenómeno.

La suplementación con lípidos está asociada en forma positiva (Giraldo y Uribe 2012); no obstante, el nivel de suplementación de grasa "by pass" es variable, y no debe exceder el $4 \%$ de la ración (Herrera et al., 2012; Tyagi et al., 2010; Salas et al., 2011). Esta suplementación incrementa la densidad energética de la dieta, mejorando el comportamiento productivo y reproductivo (Giraldo y Uribe, 2012; Hernández y Díaz 2011; Herrera et al., 2012; Tinoco y Orozco 2012).

Las grasas "by pass" consisten en una fuente de ácidos grasos insaturados, siendo los más importantes los ácidos grasos linolénico y linoléico (Duque et al., 2011), y que al estar protegidos no son utilizados por los microrganismos ruminales (Duque et al., 2011). Los ácidos grasos libres (NEFA) circulantes son metabolizados por tres vías: oxidados por el 
hígado y músculo esquelético; experimentan $\beta$-oxidación y sufren una re-esterificación a triglicéridos en el hígado; o son usados por la glándula mamaria (Rivas et al., 2011; Bobe et al., 2004). La formación de cuerpos cetónicos es directamente proporcional a la oxidación de los ácidos grasos (Zeoula y Ferreira, 2001); mientras la restricción energética no tiene efecto sobre la lipolisis en la lactación temprana (Wadhwa, et al., 2012).

Se plantea que los mayores y mejores efectos en la utilización de grasas "by pass" se logran en el primer tercio de lactancia, relacionados con una mejor utilización de la energía y no con un aumento de la oferta de energía (Giraldo y Uribe 2012), existiendo a la vez efectos sobre el eje hipotálamo-hipófisis-ovario (Staples et al. 1998), secreción hormonal, actividad ovárica (Herrera et al., 2011), consumo voluntario (García 2012) y sobre la producción (Martínez et al., 2010).

La saturación de los ácidos grasos polinsaturados (PUFA) en el rumen, ocurre como mecanismos de defensa de los microrganismos ruminales, disminuye la toxicidad de los mismos para la microbiota ruminal (Angel 2009). Cuando se utiliza en la dieta de los rumiantes una fuente de grasa activa (no sobrepasante) con altos niveles de PUFA, la mayoría de estos elementos se pierden por acción de la biohidrogenacion, limitando su utilización (Hernández y Díaz 2011; Salvador et al., 2011).

El presente estudio evaluó el efecto de la adición de diferentes porcentajes de grasa "by pass", como suplemento energético en la actividad ovárica y retorno a la ciclicidad de vacas lecheras mestizas de una propiedad típica de la parroquia Victoria del Portete (Cuenca).

\section{MATERIAL Y MÉTODOS}

El presente trabajo investigativo se llevó a cabo en la Hacienda "La Rosa" ubicada a 2850 m.s.n.m., se utilizaron 60 hembras bovinas adultas de 2 a 5 partos, con condición corporal al parto de 3 a 3.5 , sin aparentes problemas reproductivos ni metabólicos al parto; bajo un sistema de pastoreo en 30 hectáreas de una mezcla forrajera de Lolium perenne, Dactylis glomerata, Trifolium repens, Trifolium pratense; Pennisetum clandestinum y Holcus Lanatus (ceniza: 10\%; extracto etéreo: $2.82 \%$; proteína: $16.36 \%$ y fibra: $22.16 \%$; con un requerimiento de energía calculado para vacas con un peso estimado de $600 \mathrm{Kg}$, energía neta de lactancia y mantenimiento $19.4 \mathrm{Mcal}$, gasto en movilización $4.02 \mathrm{Mcal}$ y gasto en regular la temperatura 5.4 Mcal. Los animales fueron alimentados con grasa "by pass": Toco $\mathrm{BP} \circledast$ (La Fabril S.A) mezclado en 2 kg/vaca/día de concentrado Proganado Súper lechero ${ }^{\circledR}$ (proteína: $14 \%$; grasa: $3 \%$; fuente: pronaca) y suplementados con sal mineral (Ca $20 \%$ y P10\%).

La cantidad de grasa "by pass" que se les suministró fue calculada de acuerdo a los tratamientos planteados y según el intervalo de pesos, previa a la obtención del promedio y desviación estándar del grupo de animales intervinientes en el experimento, tabla 1. Se 
instauró un periodo de acostumbramiento a la grasa "by pass" (5 días antes) y se mantuvo la alimentación en los animales hasta el día 60 posparto.

Los Tratamientos se dividieron en dosis en distintos porcentajes (1, 2 y $3 \%$ ) de grasa de sobrepaso, suministradas dos veces al día. Las variables dependientes fueron: actividad ovárica (medidas a intervalos de 10 días desde el parto hasta los 60 días posparto), presencia folículos, cuerpos lúteos, número total de folículos encontrados por animal y tamaño folicular (ecografía transrectal "Sonoescape A6"), producción (litros diarios) y condición corporal (escala 1 a 5).

Se usó el paquete estadístico INFOSTAT (2011) para determinar las diferencias entre tratamientos; se los comparó con una prueba de Chi cuadrado y se utilizó estadísticas descriptivas para representar estas diferencias.

\section{RESULTADOS}

El comportamiento folicular de los animales fue analizado de acuerdo a la actividad ovárica y la presencia de estructuras ováricas presentes durante el estudio, en donde existe influencia de la grasa "by pass" en la actividad cíclica de los animales $(\mathrm{P}<0.05)$ y sobre los diferentes tipos de estructuras ováricas $(\mathrm{P}<0.05)$, tabla 2 .

\section{Variables Reproductivas}

La actividad ovárica se incrementa a partir del día 30 postparto, debido al aumento de estructuras ováricas, resumidas en la tabla 3; donde no existen diferencias estadísticas en la actividad cíclica $(P>0.05)$, debido al número de días; mientras en el efecto de la grasa "by pass", la diferencia es significativa. En lo que respecta a la presencia o ausencia de estructuras ováricas $(\mathrm{P}<0.01)$, la actividad ovárica y presencia de estructuras ováricas es significativamente mayor $(P<0.01)$, de acuerdo a la ubicación del cuerno uterino.

La actividad ovárica fue definida mediante la presencia o ausencia de cuerpos lúteos y folículos como indicadores de actividad ovárica. En este caso, tanto el tratamiento GB0\% y GB3\% muestran un mayor porcentaje de ausencia de estructuras ováricas en proporción, siendo esta diferencia más marcada en el cuerno derecho. La presencia de folículos

Tabla 1. Energía Neta de Lactancia (ENL) suministrada a cada grupo de vacas mediante suministro de grasa "by pass".

\begin{tabular}{ccccc}
\hline Grupos/\# animales & Rango & Gramos & Cantidad de ENL & Unidad \\
\hline \multirow{2}{*}{$3 \%(15)$} & Mínimo & 538 & 2.37 & \\
& Máximo & 475 & 2.10 & \\
\multirow{2}{*}{$2 \%(15)$} & Mínimo & 361 & 1.59 & \\
& Máximo & 296 & 1.31 & Mega calorías \\
& Mínimo & 175 & 0.77 & \\
$0 \%(15)$ & Máximo & 154 & 0.68 & \\
& - & 0 & 0.00 & \\
\hline
\end{tabular}


(estructuras ováricas) es mayor en los tratamientos GB1\% y GB2\%. Estos valores guardan significancia en el cuerno derecho sobre el izquierdo, demostrando mayor actividad ovárica en esta zona. Existen a la vez un mayor número de cuerpo lúteos en el tratamiento GB1\%.

Tabla 2. Porcentajes de animales que presentaron actividad ovárica.

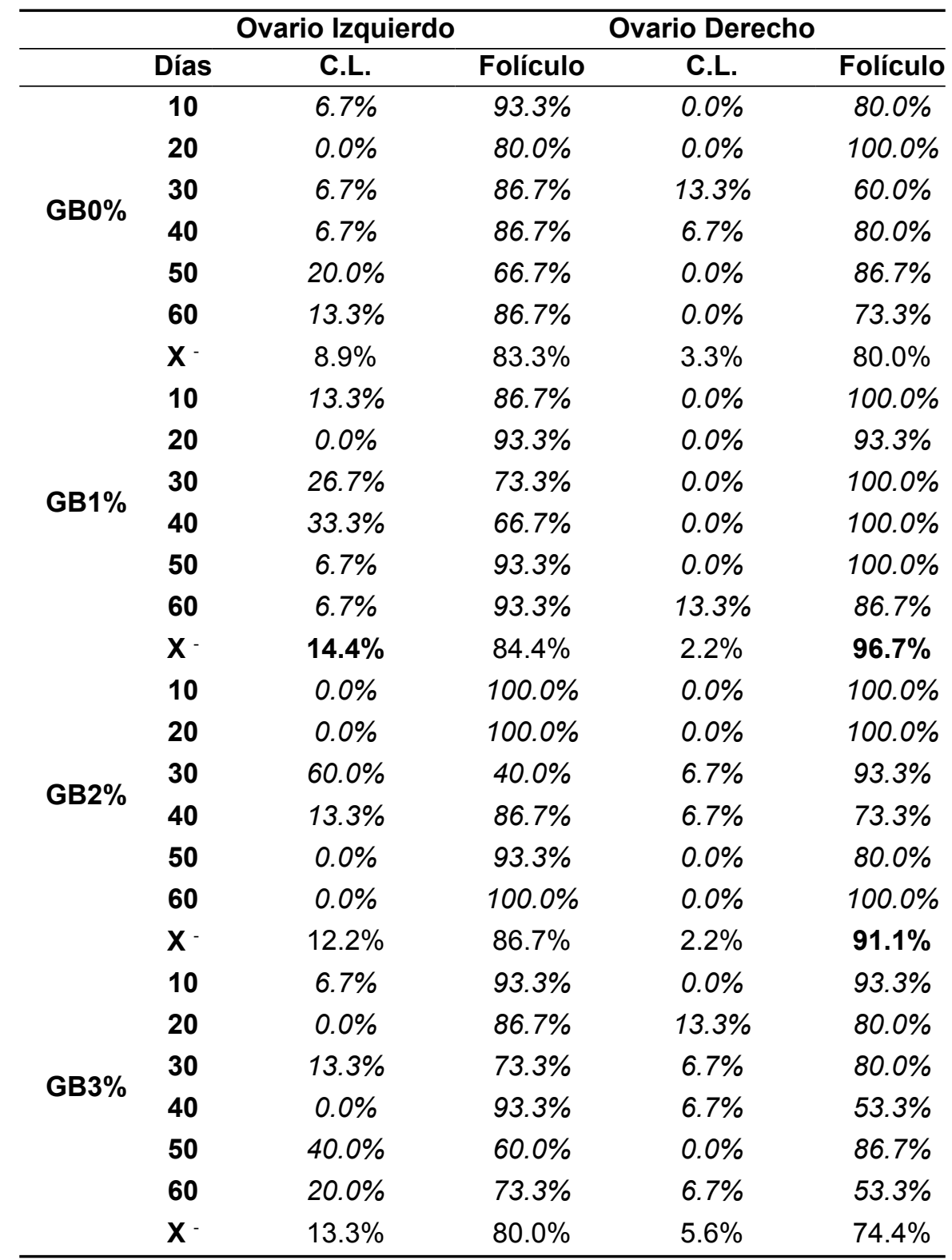

Tablas 3. Animales con actividad cíclica en relación con el Cuerpo Lúteo.

\begin{tabular}{ccccc}
\hline Días & GB0\% & GB1\% & GB2\% & GB3\% \\
\hline $\mathbf{1 0}$ & $3 \%$ & $7 \%$ & $0.0 \%$ & $3 \%$ \\
$\mathbf{2 0}$ & $0.0 \%$ & $0.0 \%$ & $0.0 \%$ & $7 \%$ \\
$\mathbf{3 0}$ & $10 \%$ & $10 \%$ & $33 \%$ & $10 \%$ \\
$\mathbf{4 0}$ & $10 \%$ & $17 \%$ & $7 \%$ & $3 \%$ \\
$\mathbf{5 0}$ & $10 \%$ & $3 \%$ & $10 \%$ & $20 \%$ \\
$\mathbf{6 0}$ & $7 \%$ & $10 \%$ & $7 \%$ & $13 \%$ \\
$\mathbf{X}-$ & $\mathbf{6 . 6 \%}$ & $\mathbf{7 . 8 \%}$ & $\mathbf{9 . 5 \%}$ & $\mathbf{9 . 3} \%$ \\
\hline
\end{tabular}


Al comparar el efecto de los días en el ciclo ovárico, se puede evidenciar el incremento de la misma a partir de los 30 días, destacándose los tratamientos GB1\% y GB\%2. En el porcentaje de animales que presentan actividad folicular, el tratamiento testigo (GB0\%) presenta valores inferiores de presencia de cuerpo lúteo $\left(37 \%^{a}\right)$ en los porcentajes acumulados $(\mathrm{P}<0.05)$, frente a los tratamientos GB1\% $\left(50 \%{ }^{\mathrm{b}}\right)$; GB2\% $\left(43 \%^{\mathrm{b}}\right)$ y GB3\% $\left(56 \%^{\mathrm{b}}\right)$ respectivamente.

Los tratamientos no afectan $(P>0.05)$ el número de folículos, donde el valor máximo fue 6.37 (GB2\%) y el mínimo de 5.61 (GB1\%). El tamaño de los folículos tampoco presenta diferencias estadísticas ( $P>0.05)$, donde el valor máximo fue de $7.43 \mathrm{~mm}(\mathrm{~GB} \% 2)$ y $6.68 \mathrm{~mm}$ (GB\%3). En lo que respecta al número de folículos y tamaño de los mismos, se encontraron diferencias $(P<0.05)$ entre los distintos días del estudio. El número de folículos no tiene diferencias estadísticas $(P<0.05)$ en relación al cuerno izquierdo $\left(5.39^{a}\right)$ y derecho $\left(6.8^{\mathrm{a}}\right)$. En lo que respecta a los análisis multifactoriales, se puede afirmar que los días postparto afecta $(p<0.05)$ en general a todos los tratamientos.

También se procedió a analizar la correlación que tienen las variables de ciclicidad $\left(P<0.05^{*}\right.$ y $\left.\mathrm{P}<0.01^{* *}\right)$ entre sí; donde a mayor tamaño, menor el número de folículos $\left(-0.39^{* *}\right)$; a la vez que conforme pasan los días postparto, el número de folículos tiende a disminuir $\left(-0.19^{* \star}\right)$, mientras el tamaño aumenta $\left(0.09^{*}\right)$.

\section{Variables de Condición Corporal y Productivas}

La condición corporal (C.C) se ve afectada por la grasa "by pass" donde el testigo (GB0\%) tuvo un promedio de $2.60^{\mathrm{a}}$; mientras los tratamientos tuvieron un promedio de $2.97^{\mathrm{b}}$ (GB\%1), $2.98^{\mathrm{b}}(\mathrm{GB} \% 2)$ y $2.99^{\mathrm{b}}$ (GB\%3) respectivamente; valores que respaldan lo citado ya por Roche et al., (2009) y respaldados por un meta-análisis realizado por Rodney et al. (2015); guardando una relación positiva $\left(0.10^{*}\right)$ con los días posparto.

Finalmente se procedió a analizar la influencia de grasa by-pass en la producción, donde la inclusión de la misma influye significativamente en la producción, tabla 4.

\section{DISCUSIÓN}

Los datos son similares a los resultados obtenidos por Tyagi et al. (2010) y los de Staples et al. (1998), quienes determinaron en varios estudios que existe una tendencia a mejorar las tasas de concepción con el empleando de grasa (jabones de calcio, aceite de pescado y sebo). Al aumentar la disponibilidad de ácidos grasos para ser absorbidos en el intestino de

Tabla 4. Efecto de la grasa by-pass sobre la producción $(P=0.05)$

\begin{tabular}{ccc}
\hline TRATAMIENTO & PRODUCCIÓN PROMEDIO & \%CV \\
\hline GBP0\% & $15.16^{\mathrm{a}}$ & $8.4 \%$ \\
GBP1\% & $18.15^{\mathrm{b}}$ & $8.1 \%$ \\
GBP2\% & $18.31^{\mathrm{b}}$ & $7.4 \%$ \\
GBP3\% & $19.11^{\mathrm{b}}$ & $10.1 \%$ \\
\hline
\end{tabular}


rumiantes, estos ácidos pueden alcanzar con mayor facilidad los tejidos reproductivos y mejorar la fertilidad (Ortega et al., 2012).

En esta investigación no se pudo evidenciar el efecto estadístico de la grasa "by pass" en el incremento del número de folículos de los tratamientos con promedios que van de $6.23^{\mathrm{a}}$ a $6.37^{\mathrm{a}}$, y promedios del control de 5.61a; siendo estos valores significantemente inferiores a los de dicho estudio, lo que se relaciona con diferentes condiciones de manejo y genéticas de este estudio. Takahashi et al., (2013), por su parte obtuvo un mayor número de folículos $\left(14.9^{\mathrm{c}} \pm 8.9\right)$ frente al control $\left(10.0^{\mathrm{b}} \pm 7.6\right)$, tomado en cuenta que el estudio mencionado se enfocó además en otros factores.

El análisis estadístico de la actividad ovárica (presencia de estructuras ováricas), en este estudio obtuvo diferencias significativas para la ubicación (cuerno), donde existe mayor actividad. Los animales tratados con grasa "by pass" (GB1\% y GB2\%), presentaron una mayor actividad específica en esta ubicación que en el grupo control, (GB0\%=7.8\%) y el tratamiento de mayor porcentaje $(\mathrm{GB} 3 \%=6.7 \%)$; confirmando que las dietas muy altas pueden alterar la ciclicidad ovárica, Duque et al. (2013), tanto como las bajas Ramteke, et al. (2014).

Los resultados a favor de dietas al 1\% de suplementación grasa en base a la ingesta de materia seca, se debe a que los lípidos ofrecidos están protegidos, reduciendo el impacto de la acción del rumen (Hernández y Díaz 2011; Rhodes et al., 2003; García, 2012).

Beam et al. (1997), determinó que las raciones de alto contenido energético incrementan el diámetro de los folículos. El tamaño de los folículos en este estudio no fue significativo entre tratamientos, con un valor máximo $(7.41 \mathrm{~mm})$ del GB2\%; valores que son numéricamente superiores a los otros estudiados.

Beam et al. (1997), señalan que hasta los 42 días posparto en vacas de alta producción; mientras en este estudio el pico de la ovulación cuantificado por la actividad folicular, se logra a partir de los 30 días, obteniéndose valores estadísticamente superiores en lo tratamientos intermedios (GB1\% y GB2\%).

Para mejorar la función reproductiva de las vacas tipo lechero a través de la suplementación lipídica, es necesario que se suministre lípidos que contengan ácidos grasos esenciales (ácidos grasos poli-insaturados o PUFAs: oleico, linoleico, linolénico) y estar protegidos para no ser afectados por la acción ruminal, ni alterar la degradación de la celulosa presente en los forrajes (Hernández y Díaz 2011).

Cuando el requerimiento de energía es mayor que su suministro, la tasa de lipolisis es superior a la lipogénesis, por lo que la vaca pierde condición corporal e incrementa los niveles séricos de ácidos grasos no esterificados (NEFA) (Roche et al., 2009). Los cambios de C.C. de este estudio confirman la acción de la grasa "by pass" donde la ingesta de 
energía metabolizable aumenta las reservas energéticas y la condición corporal (ㅂills et al., $\underline{2015)}$.

\section{CONCLUSIÓN}

Cuando se maneja en dosis recomendadas el uso de grasa "by pass" tiene efectos positivos en la actividad ovárica (tiempo), y presencia de estructuras ováricas (número) de los animales, a pesar de que los animales no sean altamente productores; por lo que tratamientos intermedios de grasa "by pass" (GB\%1 y GB\%2) obtienen mejores resultados reproductivos que los grupos control y GB\%3.

\section{LITERATURA CITADA}

ANGEL, Juan Camilo. 2009. Suplementação de vacas receptoras de embriões com ácidos graxos poliinsaturados esteríficados. Tesis de Licenciatura. Facultad de Agronomía. Universidad Federal de Rio Grande del Sur. Brasil. Pp. 107. https://www.lume.ufrgs.br/ bitstream/handle/10183/28303/000768628.pdf?sequence=1

BÁEZ G, Grajales H. 2009. Anestro Posparto en Ganado Bovino en el Trópico. Revista MVZ Córdoba. 14(3):1867-1875, 2009 ISSN: 0122-0268 https://revistas.unicordoba.edu.co/ index.php/revistamvz/article/view/347/415

BEAM Stephen, Butler WR. 1997. Energy balance and ovarian follicle development prior to the first ovulation postpartum in dairy cows receiving three levels of dietary fat. Biology of reproduction. 56:133-142. 1997. http://www.biolreprod.org/content/56/1/133.long

BECERRA JJ, Quintela L A, Díaz C, Rey C, Gracia S, Herradón PG. 2008. Estudio del Posparto en la raza bovina Rubia Gallega. Información Técnica Economica Agraria ITEA. 104 (4): 421-432. ISSN 1699-6887

BOBE G, Young JW, Beitz DC. 2004. Invited review: pathology, etiology, prevention, and treatment of fatty liver in dairy cows. Journal Dairy Science. 87: 3105-3124. http:// www.journalofdairyscience.org/article/S00220302\%2804\%2973446-3/abstract DOI:10.3168/ jds.S0022-0302(04)73446-3

BRASAEMLE D. 2013. Perilipin 5 putting the brakes on lipolysis. Journal of Lipid Research. 54: 876-877. http://www.jlr.org/content/54/4/876.full.pdf+html DOI 10.1194/jlr.E036962

DE NAVA G, Olarte C, Frade S, Reyes L, Cavestany D. 2012. Anestro posparto en vacas de cría. Resultados de la adopción de tecnologías asociadas a inseminación artificial a tiempo fijo o monta natural. Montevideo. 48:142-149. ISSN 0376-4362. http:// www.revistasmvu.com.uy/revistas/congreso-aupa.pdf

DUQUE M, Rosero R. 2011. Metabolismo energético en vacas durante la lactancia temprana y el efecto de la suplementación con grasa protegida. Revista Colombiana de Ciencias Pecuarias. 32:74-82. ISSN: 0120-0690. http://rccp.udea.edu.co 
DUQUE M, Ricardo R, Jorge G, Martha O. 2013 Efecto de la suplementación con grasas protegidas sobre parámetros productivos y reproductivos en vacas lactantes. Revista de Medicina Veterinarias y Zootecnia de Córdoba. 18(3):3812-3821. ISSN: 0122-0268 http:// www.redalyc.org/articulo.oa?id=69329149010

ESPINOZA JL, Ortega R, Palacios A, Guillén A. 2010. Efecto de la suplementación de grasas sobre características productivas, tasas de preñez y algunos metabolitos de los lípidos en vacas para carne en pastoreo. Archivo de Medicina Veterinaria. 42: 25-32 ISSN 0301-732X

http://www.scielo.cl/scielo.php? pid $=S 0301732 \times 2010000100004 \&$ script=sci arttext

GARCÍA K. 2012. Respuesta a la suplementacion con grasa sobrepasante en vacas mestizas en posparto en condiciones de trópico. Tesis de Maestria. Facultad de Ciencias Agropecuarias. Universidad Nacional de Colombia. Colombia. Pp. 64 . http:// www.bdigital.unal.edu.co/7136/1/206518.2012.pdf

GIRALDO D, Uribe L. 2012. Estrategias para mejorar la condición corporal posparto en vacas de carne. Biosalud. 11(1):19 ISSN 1657-9550 http://www.scielo.org.co/scielo.php? script $=$ sci_arttext\&pid $=$ S165795502012000100008

HERNÁNDEZ R, Thaís D. 2011. "Las grasas sobrepasantes y su efecto sobre la actividad productiva y reproductiva en rumiantes". En: González C, Madrid M, Soto E. Innovación y Tecnología en la Ganadería Doble Propósito. Pp.333-343. Astro Data S.A. ISBN 978-980-6863-10-1

HERRERA J, Tinoco J, Orozco K. 2012. Suplementación Grasa y su Efecto sobre la Reproducción de Rumiantes. Reunión Bianual sobre Reproducción Animal. UAEM-UAT. México. Pp. 17-36. https://bib.irb.hr/datoteka/598856.Compendio.pdf

HERRERA J, Soberano A, Orozco K, Aguilar C, Ku JC. 2011. "Effect of Fatty Acids on Reproductive Performance of Rumiantes". En: Milad Manafi. Artificial Insemination in Farm Animals. India. INTECH. Pp. 300. ISBN: 978-953-307-312-5

HILLS JL, Wales WJ, Dunshea FR, Garcia SC, Roche JR. 2015. An evaluation of the likely effects of individualized feeding of concentrate supplements to pasture-based dairy cows. Journal Dairy Science . 98(3):1363-1401. http://dx.doi.org/10.3168/jds.2014-8475

KAWASHIMA C, Matsui M, Shimizu T, Kida K, Miyamoto A. 2011 Nutritional factors that regulate ovulation of the dominan follicle during the firts follicular wave pospartum in highproducing dairy cows. Journal of Reproduction and Development. 58: 10-16. Disponible: http://ir.obihiro.ac.jp/dspace/bitstream/10322/4041/1/ kawashima58(1),\%2010-16,\%202012.pdf PMID: 22450279 
MARTíNEZ A, Pérez M, Pérez L, Gómez G, Garzón A. 2010. Efecto de la grasa de la dieta sobre la grasa láctea de los rumiantes: Una revisión. Interciencia. 35: 723-729.. ISSN: 0378-1844 Disponible: http://www.redalyc.org/articulo.oa?id=33915592003

MONTAÑO E, Ruiz Z. 2005. Porque no ovulan los primeros folículos dominantes de las vacas cebú posparto en el trópico colombiano. Revista Colombiana de Ciencias Pecuarias . 18 (2) 127-135. ISSN e: 2256-2958

MORALES JT, Cavestany D. 2012. Anestro posparto en vacas lecheras: tratamientos hormonales. Revista Veterinaria (Montevideo) 48 (188) 3-11 Disponible: http:// www.produccionbovina.com/produccion bovina de leche/produccion bovina leche/154anestro_19.pdf

MOTTA P, Ramos N, Gonzalez C, Castro E. 2011 Dinámica folicular en la vida reproductiva de la hembra bovina. Veterinaria y Zootécnia. 5(2): 88-99.

ORTEGA R, Espinoza J, Palacios A, Arjona O, Palacios E. 2012. Los ácidos grasos de la dieta afectan la fisiología reproductiva en la hembra bovina: una revisión.Tropical and Subtropical Agroecosystems. 15(1): 153-163. Disponible: http:// www.revista.ccba.uady.mx/ojs/index.php/TSA/article/view/1494/727

Palomares RA. 2008. Fundamentos para la terápia estratégica de las patologias posparto de la vaca. Desarrollo Sostenible de la ganadería de doble propósito. p 13 Disponible: http:// www.avpa.ula.ve/libro desarrollosost/pdf/capitulo 47.pdf

RAMTEKE PV, Patel DC, Parnerkar S, Shankhpal SS, Patel GR, Pandey A. 2014. Effect of bypass fat supplementation during prepartum and postpartum on reproductive performance in buffaloes. Livestock research international. 2: 54-58.. ISSN: 2347-5765 Disponible: http:// www.jakraya.com/Journal/pdf/5-IriArticle_3.pdf

RHODES FM, McDougall S, Burke CR, Verkerk GA, McMilla KL. 2003. Treatment of Cows with an Extended Postpartum Anestrous Interval. Journal of Dairy Science American Dairy Science Association. 86:1876-1894 http://www.journalofdairyscience.org/article/ $\underline{\text { s0022-0302(03)73775-8/pdf }}$

RIVAS C, Suárez Á, Ramírez E. 2011. Influencia de las hormonas metabólicas y la nutrición en el desarrollo folicular en el ganado bovino: implicaciones prácticas. Revista de Medicina Veterinaria- Colombia. 21: 155-173. ISSN 0122-9354. Disponible: http:// revistas.lasalle.edu.co/index.php/mv/article/view/

ROBSON CJ, Aller F, Callejas S, Alberio RH 2008. dinámica folicular posparto y comportamiento del amamantamiento en razas angus y criolla argentina. Archivos de Zootecnia. 57(220) 477-488. ISSN 0004-0592 
ROCHE JR, Friggens NC, Kay JK, Fisher MW, Stafford KJ, Berry DP. 2009 Body condition score and its association with dairy cow productivity, health, and welfare." American Dairy Science Association. Journal Dairy Science . 92: 5769-5801. Disponible: http:// www.journalofdairyscience.org/article/S00220302\%2809\%2971299-8/pdf. DOI: 10.3168/ jds.2009-2431

RODNEY RM, Celi P, Scott W, Breinhild K, Lean IJ. 2015. Effects of dietary fat on fertility of dairy cattle:A meta-analysis and meta-regression. Journal Dairy Science 98(8): 5601-5620. Disponible: http://dx.doi.org/10.3168/jds.2015-9528

RUIZ LF, Sandoval R. 2013. Involución uterina en el ganado bovino: un nuevo score para su evaluación y su relación con el número de partos y los días en lactación. Spermova.3(1):8788Disponible: $\quad$ http://www.reproduccionanimal.org/site3/files/revistas/ spermova3/87-88-20-Ruiz-Vacas involucion.pdf

SALAS GJ Herrera, Gutiérrez E, Ku J, Aké J. 2011. Reinicio de la actividad ovarica posparto y concentracion plasmática de metabolitos lípidos y progesterona en vacas suplementadas con grasa de sobrepaso.Tropical and Subtropical Agroecosystems -Redalyc. 14(2): 385-393 ISSN 1870-0462 Disponible: http://www.redalyc.org/articulo.oa?id=93918231003

SALVADOR A, Hernandez R, Díaz T, Betancourt R. 2011. Respuesta productiva y reproductiva al uso de la grasa sobrepasante con altos niveles de ácidos grasos poliinsaturados en rumiantes. Facultad de Ciencias Veterinarias, Universidad Central de Venezuela.p $19 . \quad$ Disponible: http://www.academia.edu/20588643/ respuesta_productiva_y_reproductiva_al_uso_de la_grasa_sobrepasante_con_altos_nivele s_de_\%c3\%81cidos grasos_poli-_insaturados en rumiantes

SILVA AM, Stangaferro M, Barberis F, Taboada AF, Cattaneo L, Ortega H. 2011. Factores Asociados a la reanudación de la ciclicidad ovárica posparto en vacas lecheras y de carne. Revista FAVE Ciencias Veterinarias. 10(1): 33-48 ISSN 1666-938X.

STAPLES CR, Burke JM, Thatcher WW. 1998. Influence of Supplemental Fats on Reproductive Tissues and Performance of Lactating Cows. Journal of Dairy Science. 81: 856-871. http://www.journalofdairyscience.org/article/S0022-0302\%2898\%2975644-9/pdf PMID: 9565891 DOI: 10.3168

TAKAHASHI M, Kumiku S, Kawate N, Inaba T, Tamada H. 2013. Improvement of superovulatory response and pregnancy rate after transfer of embryos recovered from japanese black cows fed rumen bypass polyunsaturated fatty acids. Theriogenology Journal Veterinary Medicine Scicience. 75 (11): 1485-1490. Disponible: https:// www.ncbi.nlm.nih.gov/pmc/articles/PMC3942974/ doi: 10.1292/jvms.12-0235 
TYAGI N, Thakur SS, Shelke. 2010. Effect of bypass fat supplementation on productive and reproductive performance in crossbred cows. Tropical Animal Health Production-Springer. 42(8): 1749-1755 ISSN 1573-7438. http://link.springer.com/article/ 10.1007\%2Fs11250-010-9631-1

WADHWA M, Grewal RS, Bakshi MP, Brar PS. 2012. Effect of supplementing bypass fat on the performance of high yielding crossbred cows. Indian Journal of Animal Sciences. 82(2): 200-203. ISSN: 0367-8318. http://epubs.icar.org.in/ejournal/index.php/IJAnS/article/view/ 15268

WILTBANK M, Gümen A, Roberto S. 2002. Physiological classification of anovulatory conditions in cattle. Theriogenology. 57(1): 21-52. https://www.researchgate.net/profile/ Roberto Sartori/publication/ 11581987 Physiological classification of anovulatory conditions in cattle/links/ 5461055e0cf2c1a63bff7aee.pdf DOI: 10.1016/S0093-691X(01)00656-2

ZEOULA LM, Ferreira S. 2001. Recentes avanços em amido na nutrição de vacas leiteiras. Edited by Julio César Teixeira, Roseli dos Santos, Flávia David, \& Lucia Andrade. Lavras: UFLA-FAEPE. 2: 199-228. Universidad Federal de Lavras. Brasil. Simposio Internacional em Bovinocultura de Leite. 